\title{
MASS DETECTION IN DIGITAL MAMMOGRAMS USING GABOR FILTER BANK
}

\author{
Muhammad Hussain ${ }^{1}$, Salabat Khan ${ }^{2}$, Ghulam Muhammad ${ }^{3}$, Mohamed Berbar ${ }^{1}$ and George Bebis ${ }^{4}$ \\ ${ }^{I}$ Department of Computer Science, ${ }^{3}$ Department of Computer Engineering; College of Computer and Information Sciences, \\ King Saud University, Riyadh 11543, Saudi Arabia \\ ${ }^{2}$ National University of Computer and Emerging Sciences, Islamabad, Pakistan \\ ${ }^{4}$ Department of Computer Science and Engineering, University of Nevada at Reno \\ \{mhussain, ghulam\}@ksu.edu.sa
}

Keywords: Breast cancer detection, Gabor filter bank, Textural properties, Feature extraction, Digital mammography

\begin{abstract}
Digital Mammograms are currently the most effective imaging modality for early detection of breast cancer but the number of false negatives and false positives is high. Mass is one type of breast lesion and the detection of masses is highly challenged problem. Almost all methods that have been proposed so far suffer from high number of false positives and false negatives. In this paper, a method for detecting true masses is presented, especially, for the reduction of false positives and false negatives. The key idea of the proposal is the use of Gabor filter banks for extracting the most representative and discriminative local spatial textural properties of masses that are present in mammograms at different orientations and scales. The system is evaluated on 512 (256 normal+256 true mass) regions of interests (ROIs) extracted from digital mammograms of DDSM database. We performed experiments with Gabor filter banks having different numbers of orientations and scales to find the best parameter setting. Using a powerful feature selection technique and support vector machines (SVM) with 10-fold cross validation, we report to achieve $\mathrm{Az}=0.995 \pm 0.011$, the area under ROC. Comparison with state-of-the-art techniques suggests that the proposed system outperforms similar methods, which are based on texture description, and the difference is statistically significant.
\end{abstract}

\section{Introduction}

Breast cancer is the most common form of cancer that affects women all over the world and is considered a major health problem. According to the statistics of National Cancer Institute, Surveillance, Epidemiology, and End Results (SEER) program, lifetime risk of developing breast cancer among American women is $12.2 \%$ (aka: one in eight), exceeded only by the lung cancer $[1,15]$. In the European Community, breast cancer represents $19 \%$ of cancer deaths and $24 \%$ of all cancer cases [9, 4]. Women diagnosed between ages $40-49$ years are the major victims having about $25 \%$ of all breast cancer deaths. The World Health Organization's International Agency for Research on Cancer (IARC) has estimated more than one million cases of breast cancer to occur annually and reported that more than 400, 000 women die each year from this disease [11].
Mammography is considered to be the most effective screening method for the detection of breast cancer. By digitizing the mammograms and exploiting contemporary powerful image analysis techniques, computer aided diagnosing (CAD) systems can be developed for effectively assisting the radiologists. There are three types of breast lesions; masses, calcifications and architectural disorders [15]. The target of this research work is to develop a method for the detection of true masses in digital mammograms.

Although, the most accurate mass detection could be done using biopsy, it is an expensive procedure and involves some risk e.g. patient discomfort, post biopsy side effects, chances of missing cancerous tissues based on different biopsy methods. On the other hand, CAD systems are easy to use tools that are inexpensive and by analysing the digital mammograms they can effectively assist the radiologists in their decision making process (as a second expert opinion).

The idea of using CAD system for breast cancer detection is not recent. CAD systems have been used earlier for this task and proved to be useful in the screening process of digital mammograms and the detection of early stage malignancies $[15,9,11]$. However, there exist controversial results and views against the usage of CAD systems mainly because of their high false positive and false negative rates in the breast cancer detection, which makes radiologist not really trust them [9]. In this research work, our motivation is to develop a CAD system that can detect the cancerous regions as accurately as possible and reduce both the false positive and false negative rates.

In this paper, we propose a novel approach for breast cancer detection, specifically, true mass detection by analysing the local textural properties of the masses. For this purpose, we use the Gabor filter bank to extract the local texture descriptors to characterize the micro-patterns (e.g. edges, lines, spots and flat areas) and preserve at the same time the spatial information of the masses and other regions in the digital mammograms. These texture properties are useful for correctly detecting true masses [9].

Gabor filters have been used for breast cancer detection earlier (e.g. see [15] and references therein); in these approaches, Gabor filters are applied on the whole image or the whole ROI for extracting features, which are normally global features. Our work proposes to apply the Gabor filter bank on different sub-regions of the ROIs extracted from mammograms and extract the moments based features from the magnitude Gabor responses; these features are robust against noise and represent micro-patterns occurring at different scales, orientations and locations in masses in a 
better way and give remarkable results as compared to the previous techniques. The main contributions of our work are as follows.

- A mass detection method that is equipped with the scale and rotation invariant local texture descriptors and is robust against noisy data.

- An efficient feature extraction method which can be fully parallelized in order to achieve a better computational throughput.

The remainder of this paper is organized as follows. In Section 2, we present the proposed method. Subsequently, in Section 3, we present some experimental results to show the effectiveness of the proposed technique. Finally, Section 4 concludes this work.

\section{Methodology}

In this section, we discuss all the stages of our method for breast cancer detection. First, a brief overview of Gabor Filter bank is given for a deep understanding of the subsequent processing tasks such as partition of ROIs and feature extraction. Afterwards, we give an overview of the support vector machine used for classifying ROIs as normal or cancer regions. The most computationally intensive task in our CAD system is the feature extraction procedure. Feature extraction procedure includes: 1) the partitioning of ROIs into subregions (sub-windows) ,2) application of Gabor filter bank on each sub-region, separately, and 3) collecting moments (mean, standard deviation, skewness) based features from the magnitude of Gabor filter bank responses. Fortunately, feature extraction task can be highly parallelized to achieve the better computational efficiency. This parallelization can be achieved at three levels, first at individual ROI level, second at ROI sub-regions level and third at Gabor filter processing level. In our experiments, we have not used any level of parallelization.

\subsection{Gabor Filters Bank}

Texture is an important part of the visual world of animals and humans; they can successfully detect, discriminate, and segment texture using their visual systems [12]. Textural properties in an image can be used to collect different kinds of information e.g. micro-patterns like edges, lines, spots \& flat areas. Masses in mammograms do contain edges and local spatial patterns at different scales and orientations. These micro-patterns are helpful in the detection of cancerous regions with a CAD system. Gabor filters can effectively be used to detect these micro-patterns and this research work aims to validate this statement. A brief overview of the Gabor filters is given in the next paragraphs.

Gabor filters are biologically motivated convolution kernels that have enjoyed wide usage in a myriad of applications in the field of computer vision and image processing e.g. face recognition [16], vehicle detection [17] etc. In order to extract local spatial textural micro-patterns in mammogram ROIs, Gabor filters can be tuned with different orientations and scales, and thus provide powerful statistics which could be very useful for breast cancer detection.
A two-dimensional Gabor filter defined as a Gaussian kernel modulated by an oriented complex sinusoidal wave can be described as follows [17]:

$$
\begin{gathered}
g(x, y)=\frac{1}{2 \pi \sigma_{x} \sigma_{y}} e^{-\frac{1}{2}\left(\frac{\tilde{x}^{2}}{\sigma_{x}^{2}}+\frac{\tilde{y}^{2}}{\sigma^{2}}\right)} e^{2 \pi j W \tilde{x}}, . \\
\tilde{x}=x \cdot \cos \theta+y \cdot \sin \theta \quad \text { and } \quad \tilde{y}=-x \cdot \sin \theta+y \cdot \cos \theta .
\end{gathered}
$$

where $\sigma_{x}$ and $\sigma_{y}$ are the scaling parameters of the filter and describe the neighborhood of a pixel where weighted summation takes place, $W$ is the central frequency of the complex sinusoidal and $\theta \in[0, \pi)$ is the orientation of the normal to the parallel stripes of the Gabor function. A particular bank of Gabor filters contain multiple individual Gabor filters adjusted with different parameters (scaling, orientation and central frequency). In this paper, different Gabor filter banks are used e.g. a Gabor filter bank containing 6 filters $(2$ scales $\{\mathrm{S}\} \times 3$ orientations $\{\mathrm{O}\}), 15$ filters $(3 \mathrm{~S} \times 5$ $\mathrm{O}), 24$ filters $(4 \mathrm{~S} \times 6 \mathrm{O})$ and 40 filters $(5 \mathrm{~S} \times 8 \mathrm{O})$ with initial maximum frequency equal to 0.2 and initial orientation set to 0 .

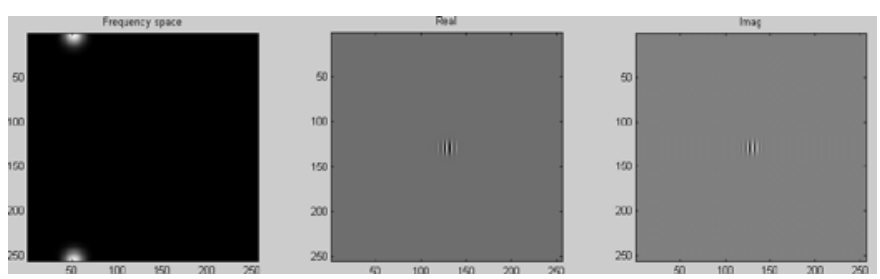

Figure 1. Gabor Filter with frequency $=0.2$, orientation $=$ 0 degree.

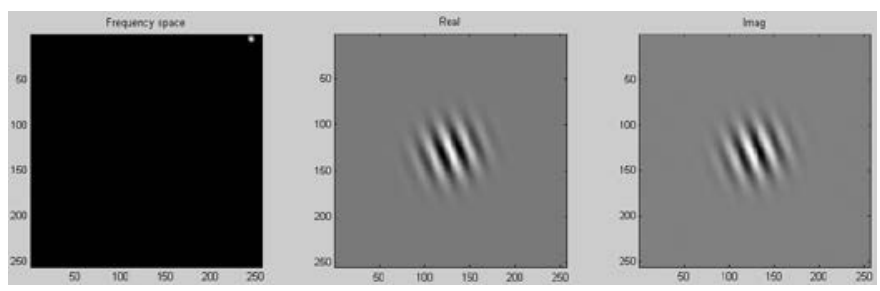

Figure 2. Gabor Filter with frequency $=\mathbf{0 . 0 5}$, orientation $=157.5$ degree.

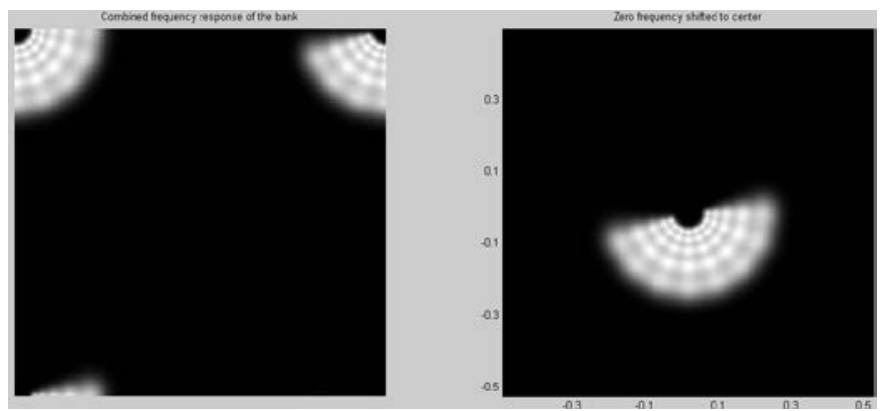

Figure 3. Combined frequency response of the 40 filters in the Gabor bank.

The orientations and frequencies for a bank are calculated using the following equations: 


$$
\begin{aligned}
& \text { orientation }(i)=\frac{(i-1) \pi}{m} \text { where } i=1,2, \ldots, m, \\
& \text { frequency }(i)=\frac{f_{\max =0.2}}{(\sqrt{2})^{i-1}} \quad \text { where } i=1,2, \ldots \ldots, n,
\end{aligned}
$$

where $m$ is the total number of orientations and $n$ is the total number of frequencies. For instance, the bank with 5 scales and 8 orientations has the frequencies $(0.2,0.14,0.1,0.07$ and $0.05)$ for a given number of scales and orientation angles $(0$, 22.5, 45, 67, 90, 112.5, 135 and 157 in degrees). In Figures 1 and 2 , two filters from this bank with $\left(f=0.2, \mathrm{o}=0^{\circ}\right)$ and $(f=$ $\left.0.05, \mathrm{o}=157.5^{\circ}\right)$ are shown for an image with resolution $256 \times 256$. Figure 4 plots the combine frequency response of the entire 40 filters in this bank.

\subsection{Partition of ROIs}

Once the ROIs are extracted from the mammograms, next step is to partition an ROI in different parts such that the features, representing local spatial texture description, can easily be extracted. Instead of applying a Gabor filter bank to the entire ROI, an ROI is first partitioned into sub-windows in our method. In particular, each ROI of size $512 \times 512$ pixels is first divided into square patches of equal size e.g. $128 \times 128$ pixels; as depicted with squares enclosed with black lines in Figures 4. In this way, sixteen patches are created, labelled 116 in Figure 4. The patches are then combined to create overlapping sub-windows e.g. patches $1,2,5$ and 6 comprise the first $256 \times 256$ pixels sub-window, $2,3,6$ and 7 the second, $5,6,9$ and 10 the fourth, and so on. With this formation, 9 overlapping sub-windows are created. First sub-window is visualized with transparent red colour rectangle in Figure 4. It may please be noted that by increasing/ decreasing the size of a patch, ROI image can be partitioned in different sizes and numbers of sub-windows.

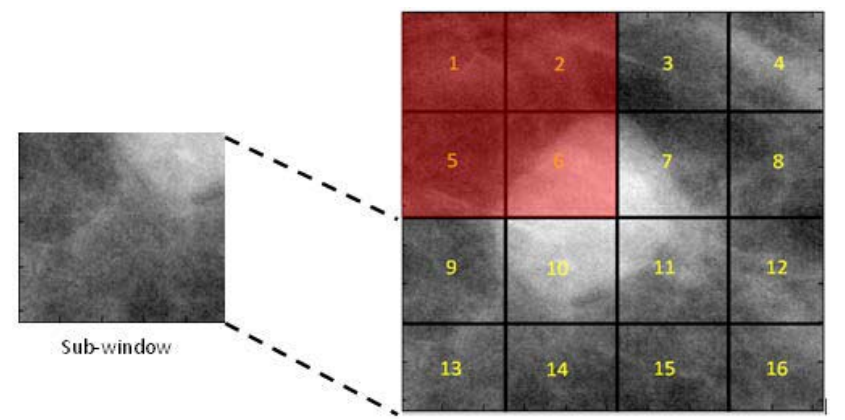

Figure 4. Segmentation of ROI in patches and subwindows

\subsection{Feature Extraction}

Feature extraction is performed by convolving sub-windows with a Gabor filter bank. A slightly modified design strategy has already been used for texture based feature extraction for vehicle detection [17] and image retrieval [10]. The raw responses of Gabor filter bank can be used as features for classification but usually some preprocessing is performed to acquire most representative features e.g. Gabor energy and moments of Gabor filter bank responses $[17,5]$. Similar to the approach given in [17] for vehicle detection, in this paper, magnitude response of each Gabor filter in the bank is collected from each sub-window and is represented by three moments: the mean $\mu_{i, j}$, the standard deviation $\sigma_{i, j}$ and the skewness $k_{i, j}$ (where $i$ corresponds to the $i$ th filter in the bank and $j$ to the $j$ th sub-window). These moments correspond to the statistical properties of a group of pixels in a sub-window and positioning of pixels is essentially discarded which compensates for any errors that might occur during extraction/ segmentation of ROIs into sub-windows. Suppose, we are using a Gabor filter bank of 40 filters $(5 \mathrm{~S} \times 8 \mathrm{O})$; applying this bank on 9 sub-windows (Figure 4) of a single ROI, yields a feature vector of length 1080. A row feature vector obtained in this way is shown below:

$$
\left(\mu_{1,1}, \sigma_{1,1}, k_{1,1}, \ldots, \mu_{40,1}, \sigma_{40,1}, k_{40,1}, \mu_{1,2}, \sigma_{1,2}, k_{1,2}, \ldots ., \mu_{40,9}, \sigma_{40,9}, k_{40,9}\right) \text {. }
$$

Table 1contains the detail of data sets based on different parameters used for feature extraction from ROIs each of size $512 \times 512$ pixels. For further reference, names are assigned to data sets.

\begin{tabular}{|c|c|c|c|c|c|}
\hline P. Size & Sub-Windows & Data set & Scales & Orientations & \#Features \\
\hline \multirow{4}{*}{$128 \times 128$} & \multirow{4}{*}{9} & D-1 & 2 & 3 & 162 \\
\hline & & D-2 & 3 & 5 & 405 \\
\hline & & D-3 & 4 & 6 & 648 \\
\hline & & D-4 & 5 & 8 & 1080 \\
\hline \multirow{4}{*}{$64 \times 64$} & \multirow{4}{*}{49} & D-5 & 2 & 3 & 882 \\
\hline & & D-6 & 3 & 5 & 2205 \\
\hline & & D-7 & 4 & 6 & 3528 \\
\hline & & D-8 & 5 & 8 & 5880 \\
\hline \multirow{4}{*}{$32 \times 32$} & \multirow{4}{*}{225} & D-9 & 2 & 3 & 4050 \\
\hline & & D-10 & 3 & 5 & 10125 \\
\hline & & D-11 & 4 & 6 & 16200 \\
\hline & & D-12 & 5 & 8 & 27000 \\
\hline
\end{tabular}

\section{Table 1. Parameters used for feature extraction sing Gabor Filter Banks}

\subsection{Classification}

A classification problem encompasses the assignment of an unseen pattern to a predefined class, according to the characteristics of the pattern, presented in the form of a feature vector. However, a classifier is first needed to be trained in order to perform this task. The training is performed using selected patterns of the same concept class, also called training set. Numerous classification techniques exist. We used SVM for the classification of ROIs. In our case, we are dealing with a binary classification problem, where an ROI can be classified either cancer region or normal.

SVM classifiers [13] are the most advanced ones, generally, designed to solve binary classification problems; thus perfectly suite our requirements. SVM finds an optimal hyper-plane that can separate the data belonging to different classes with large margins in high dimensional space [2]. The margin is defined as the sum of distances to the decision boundary (hyper-plane) from the nearest points (support vectors) of the two classes. SVM formulation is based on statistical learning theory and has attractive generalization 
capabilities in linear as well as non-linear decision problems $[13,3]$. SVM uses structural risk minimization as opposed to empirical risk minimization [13] by reducing the probability of misclassifying an unseen pattern drawn randomly from a fixed but unknown distribution.

Let $D=\left\{\left(x_{i}, y_{i}\right)\right\}_{i=1}^{N} \subset \square^{J} \times\{+1,-1\}$ be a training set where $x_{i}$ is the $i$ th training instance containing $J$ features, $y_{i}$ is the class label of $x_{i}$ having two values $\{+1$ or -1$\}$. Finding an optimal hyper-plane based on large margin framework implies solving a constrained optimization problem using quadratic programming and can be stated as:

$$
f(x)=\sum_{i=1}^{N} \alpha_{i} y_{i} k\left(x_{i}, x\right)+b .
$$

where $\alpha_{i}>0$ are the Langrange multipliers, $k\left(x_{i}, x\right)$ is the kernel function and sign of $f(x)$ gives the membership class of $x$. For linearly separable problems or linear SVM, kernel function is simply the dot product of the two given points in the input space. However, for non-linear SVMs, the original input space is mapped to the higher dimensional space through a non-linear mapping function, possibly making the data linearly separable, using different suitable kernels (for computational efficiency) defined as a dot product in the new space and satisfies the Mercer's condition [13]. In this new formulation, the misclassification penalty or error is controlled with a user defined parameter $C$ (regularization parameter, controlling trade-off between error of SVM and margin maximization), and is tied with the kernel. There are several kernels to be used e.g. linear, polynomial, sigmoid, radial basis function (RBF) etc. In our experiments, RBF kernel is used as given by:

$$
k\left(x_{i}, x\right)=\exp \left(-\gamma\left\|x_{i}-x\right\|^{2}\right), \gamma>0 .
$$

The $\gamma$ is the width of the kernel function. There are two parameters now tied with the RBF kernel: $\gamma$ and $C$. Tuning these parameters in an attempt to find a better hypothesis is called model selection procedure. For model selection, we first perform a loose grid search (coarse search for computational efficiency) to find the better region in the parameter space. Later, the finer grid search is conducted in the region found by loose grid search. This model selection procedure is recommended in the work of Chih-Wei Hsu et. al. [6]. The selected parameters are fed into the kernel and SVM is finally applied to our data sets.

\subsection{Mass Detection System}

The block diagram of the cancer detection system is shown in Figure 5. There are four main components of the system: preprocessing, feature extraction, feature selection and classification. Various existing approaches differ in the choice of techniques for these components. Our proposed approach for feature extraction is robust against noise, so there is no need for pre-processing methods for denoising and enhancement. Our method for feature extraction has been described in detail in subsections 2.3 and 2.4. The method proposed by Yijun et al. [14] is used for selecting the most significant features and SVM with RBF is employed for classification.

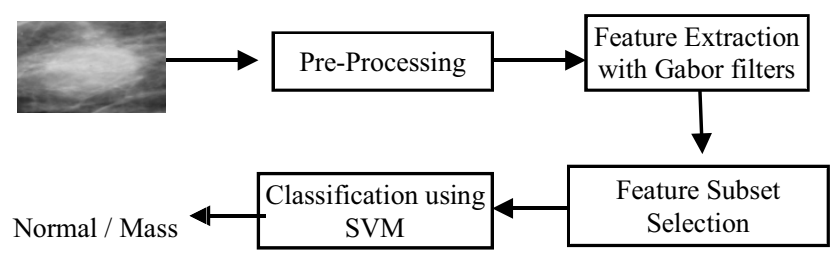

Figure 5. Mass detection system

\section{Results and Discussions}

In this section, we present the simulation results of our mass detection system. First, we discuss the database and the evaluation strategy used in our experiments. Second, the effectiveness of the number and the size of overlapping subwindows along with different Gabor Filter Bank parameter settings is evaluated. Afterwards, we give comparison of the proposed system with stat-of-the-art similar methods that exploit texture properties and address the problem of the detection of true masses $[12,8]$.

\subsection{Database and Evaluation Methodology}

The proposed method is evaluated using Digital Database for Screening Mammography (DDSM) [7]; this database consists of more than 2000 cases and is commonly used as a benchmark for testing new proposals dealing with processing and analysis of mammograms for breast cancer detection. Each case in this database is labelled by expert radiologists; the complete information is provided as an overlay file. The locations of masses in mammograms specified by experts are encoded as code-chains. We randomly selected 512 (256 normal and 256 mass) cases from the database. Using code chains, we extracted 256 ROIs which contain true masses; the sizes of these ROIs are $512 \times 512$. In addition, we extracted 256 ROIs containing normal but suspicious tissues. Some sample ROIs are shown in Figure 6. These ROIs are used for training and testing.
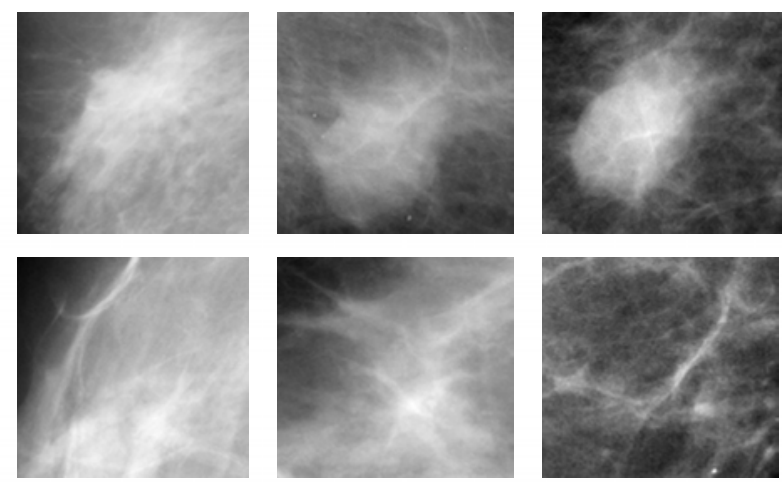

Figure 6. Sample mass ROIs (top row) and normal but suspicious ROIs (bottom row)

Commonly used evaluation measures of the predictive ability of the breast cancer detection systems are sensitivity (a measure of true positive rate), specificity (a measure of true 
negative rate), accuracy and area under ROC curve (AUC or $A z)$. We adopt these performance measures to evaluate the proposed system.

Table 2. Performance Evaluation based on varying subwindow sizes and configuration of Gabor Filters Bank (value after \pm is standerd deviation) using $\mathbf{1 0}$-fold cross validation.

\begin{tabular}{|c|c|c|c|c|}
\hline Data set & Sensitivity & Specificity & Accuracy & Az. \\
\hline D-1 & $96.43 \pm 3.96$ & $95.10 \pm 5.92$ & $95.49 \pm 3.46$ & $0.96 \pm 0.04$ \\
\hline D-2 & $97.63 \pm 2.68$ & $94.61 \pm 4.22$ & $96.07 \pm 2.26$ & $0.95 \pm 0.03$ \\
\hline D-3 & $97.23 \pm 4.14$ & $96.89 \pm 2.97$ & $96.86 \pm 2.48$ & $0.97 \pm 0.02$ \\
\hline D-4 & $97.19 \pm 2.71$ & $97.99 \pm 2.85$ & $97.64 \pm 1.80$ & $0.97 \pm 0.02$ \\
\hline D-5 & $97.18 \pm 3.45$ & $97.63 \pm 2.86$ & $97.25 \pm 2.64$ & $0.97 \pm 0.02$ \\
\hline D-6 & $97.29 \pm 3.07$ & $98.56 \pm 1.86$ & $97.84 \pm 1.71$ & $0.98 \pm 0.02$ \\
\hline D-7 & $97.95 \pm 2.92$ & $98.00 \pm 2.11$ & $98.03 \pm 1.30$ & $0.98 \pm 0.01$ \\
\hline D-8 & $\mathbf{9 8 . 0 5} \pm \mathbf{3 . 5 0}$ & $\mathbf{9 8 . 0 6} \pm \mathbf{2 . 6 8}$ & $\mathbf{9 8 . 0 3} \pm \mathbf{1 . 8 4}$ & $\mathbf{0 . 9 8} \pm \mathbf{0 . 0 1}$ \\
\hline D-9 & $96.20 \pm 3.80$ & $95.86 \pm 5.41$ & $96.07 \pm 3.81$ & $0.96 \pm 0.03$ \\
\hline D-10 & $97.34 \pm 3.17$ & $98.05 \pm 2.92$ & $97.64 \pm 2.41$ & $0.97 \pm 0.03$ \\
\hline D-11 & $97.25 \pm 4.22$ & $97.68 \pm 3.72$ & $97.45 \pm 3.46$ & $0.97 \pm 0.03$ \\
\hline D-12 & $96.67 \pm 4.05$ & $97.22 \pm 2.77$ & $97.05 \pm 1.66$ & $0.97 \pm 0.02$ \\
\hline
\end{tabular}

The evaluation of the proposed mass detection system is performed using 10 -fold cross validation. A data set is randomly partitioned into 10 non-overlapping and mutually exclusive subsets. For the experiment of fold $i$, subset $i$ is selected as testing set and the remaining subsets are used to train the classifier. Using 10 -fold cross validation, the performance of the CAD system can be confirmed against any kind of the selection bias of the samples for training and testing phases. It also helps in determining the robustness of the system when tested over different ratios of normal and abnormal ROIs used as training and testing sets (due to random selection, ratios will be different). In order to have a fair comparison, same 10-fold cross validation subsets are used for all the data sets during all the experiments. The SVM classifier gives a membership value of each class when an unseen pattern is presented to it. The ROC curve can be obtained by varying the threshold on this membership value. Afterwards, area (Az) under ROC curve can be calculated corresponding to the ROC curve.

\subsection{Results with varying sub-window sizes and Gabor bank parameters}

In order to extract features at different level of granularities, we partition the ROIs with different sizes of overlapping subwindows. The overlapping of sub-windows makes it possible to analyse those textural micro-patterns of masses that might be present at the boundaries of the sub-windows. The Gabor filter bank having multi-scale and multi-oriented filters with an initial maximum frequency and orientation is applied to each individual sub-window and three moments (mean, standard deviation and skewness) are calculated from the magnitude of Gabor filter response. Varying the size of subwindows and using a particular Gabor filter bank multiple data sets with features ranges from 162-27000 are created, as shown in Table 1. The sizes of sub-windows are dependent on the patch sizes as discussed in Section 2.2. We tested the performance of the proposed system on three different patch sizes $(128 \times 128,64 \times 64$ and $32 \times 32)$. The Gabor filter bank is initialized with initial max frequency $=0.2$, initial orientation $=0$ degree and with four different configurations of scales and orientations i.e. banks having 2 scales and 3 orientations (referred to as G23), G35, G46 and G58. This setting of Gabor filter banks can extract local textural features at different scales and orientations and gives good results as shown in Table 2. It may please be noted that we have not tried to optimize the parameter setting for Gabor Filters bank. The best Az value of $0.98 \pm 0.01$ is obtained for the data set D8 where sub-window size is $64 \times 64$ and G46 Gabor filter bank is used. For the data sets with large number of features the performance diminishes in the presence of copious redundant and irrelevant features. The results are encouraging as the system performs well even with the coarse level of feature extraction procedure i.e. moments calculation for a large group of pixels. This directly means the reduction in computation cost as few Gabor filters are applied on lesser number of sub-windows generating lower dimensional data sets and in turn SVM learning is also done in a split of minutes. However, in order to achieve better results in terms of mean $\mathrm{Az}$ value and especially lower computational cost, we performed experiments with feature selection. We applied feature subset selection (FSS) method of Yijun et at. [14] for reducing the dimension of the feature space. The results with and without feature subset selection are shown in Table 3. It is obvious that FSS reduces the dimension of the feature space and enhances the detection performance.

\section{Table 3. Detection performance with dataset $D 8$ w/o and with feature subset selection (FSS)}

\begin{tabular}{|l|c|l|c|}
\hline Dataset & \#Features & Accuracy & AUC \\
\hline with FSS & 174 & $99.60 \pm 0.82$ & $0.995 \pm 0.011$ \\
\hline w/o FSS & 5880 & $98.03 \pm 1.84$ & $0.98 \pm 0.01$ \\
\hline
\end{tabular}

\subsection{Comparison with other methods}

We compared the proposed method with two state-of-the-art methods which also exploit the texture properties of masses. One of these uses LBP descriptor (LBP method) [9] and the other uses a variant of WLD descriptor [8] - MSWLD (Multiscale Spatial WLD). We implemented these methods using the same software and hardware environment, and tested on the same database, which we used for the evaluation of our method. The comparison results are shown in Table 4. It is obvious that the proposed method performs better than LBP and MSWLD based methods. Further to test whether the classification results of the proposed method are significantly higher than those of LBP and MSWLD based methods, we used 2-tail t-test. The null hypothesis is that the difference between the mean measures of performance (accuracy and 
$\mathrm{Az}$ ) is zero and the alternative hypothesis is that the difference is positive. The test results with $5 \%$ significance level are given in Table 5. The null hypothesis is rejected at 0.05 significance level for the two methods. It indicates that the classification performance obtained using the proposed method is higher than that obtained by other two methods and the difference is statistically significant. All p-values are much less than 0.05, which indicates that the differences are actually statistically highly significant.

Table 4. Comparison between Gabor, MSWLD, and LBP

\begin{tabular}{|l|c|c|}
\hline Method & Accuracy & $\mathrm{Az}$ \\
\hline Gabor (G) & $\mathbf{9 9 . 6 0} \pm \mathbf{0 . 8 2}$ & $\mathbf{0 . 9 9 5} \pm \mathbf{0 . 0 1 1}$ \\
\hline MSWLD (M) [8] & $98.00 \pm 0.55$ & $0.988 \pm 0.006$ \\
\hline LBP (L) [9] & $92.00 \pm 0.99$ & $0.922 \pm 0.016$ \\
\hline
\end{tabular}

Table 5.Statistical significance 2-tail t-test results

\begin{tabular}{|l|l|l|l|l|}
\hline \multirow{2}{*}{ Method } & \multicolumn{2}{|l|}{ Accuracy } & \multicolumn{2}{c|}{ Az } \\
\cline { 2 - 5 } & $\mathrm{t}$-val & p-val & t-val & p-val \\
\hline G vs M & 6.87 & $2.002 \times 10^{-06}$ & 3.087 & 0.0063 \\
\hline G vs L & 21.115 & $3.76 \times 10^{-14}$ & 13.58 & $6.72 \times 10^{-11}$ \\
\hline
\end{tabular}

\section{Conclusion}

We proposed a new method for the detection of true masses from digital mammograms and to reduce the number of false positives and false negatives. The proposed method addresses the multiscale and multi-orientation textural properties of masses and represent them using Gabor filter bank. Gabor filter bank involves different parameters like scales and orientations. An approach that is robust against noise is used for extracting features; this approach requires an ROI to be divided into sub-windows. We performed experiments with different Gabor filter bank parameter settings and different sub-window sizes. For classification, we used SVM and evaluated its performance using 10 -fold cross validation. To reduce the dimension of the feature space, we employed a powerful feature selection method that significantly reduces the dimension of the feature space and improves the detection accuracy. The proposed system achieved Az $=0.995 \pm 0.011$, and accuracy $=99.60 \pm 0.82$. The comparison with sate-f-theart similar techniques that exploit textural properties of masses reveals that the proposed method outperforms and the difference is statistically significant.

Gabor filter involves four parameters: two scaling parameters, the central frequency of the complex sinusoidal and the orientation of the normal to the parallel stripes of the Gabor function. We tried a few combinations of these parameters. To choose the best combination is an optimization problem, we will address this problem in our future work.

\section{Acknowledgements}

This work is supported by the National Plan for Science and Technology, King Saud University, Riyadh, Saudi Arabia under project number 8-INF325-02.

\section{References}

[1] S. F. Altekruse, CL. Kosary, M. Krapcho, et al. "SEER Cancer Statistics Review”, 1975-2007. Bethesda, MD: National Cancer Institute, (2010).

[2] B. E. Boser, I. M. Guyon, V. Vapnik, "A training algorithm for optimal margin classifiers", In Proc. of the fifth annual workshop on Computational learning theory, pp. 144-152, (1992).

[3] C. Burges, "Tutorial on support vector machines for pattern recognition”, Data Mining Knowledge Discovery., Vol. 2, No. 2, pp. 955-974, (1998).

[4] J. Esteve, A. Kricker, J. Ferlay, D. Parkin. "Facts and figures of cancer in the European Community", In: Tech. rep., International Agency for Research on Cancer, (1993).

[5] S. Grigorescu, N. Petkov, P. Kruizinga, "Comparison of texture features based on Gabor filters", IEEE Transaction on Image Processing., Vol. 11, Issue. 10, pp. 1160-1167, (2002).

[6] C. W. Hsu, C. C. Chang, and C. J. Lin, "A Practical Guide to Support Vector Classification", Technical report, Department of Computer Science and Information Engineering, National Taiwan University, (2010).

[7] M. Heath, K. Bowyer, D. Kopans, R. Moore, P.J. Kegelmeyer "The digital database for screening mammography", Int. Work. Dig. Mammogr. pp. 212-8, (2000).

[8] M. Hussain, N. Khan, "Automatic mass detection in mammograms using multiscale spatial weber local descriptor", in Proc. IWSSIP 2012 (to appear), Austria, April 11-13, (2012).

[9] X. Lladó, A. Oliver, J. Freixenet, R. Martí, J. Martí, "A textural approach for mass false positive reduction in mammography", Computerized Medical Imaging and Graphics, Vol. 33, Issue. 6, pp. 415-422, (2009).

[10] B.S. Manjunath, W.Y. Ma., "Texture features for browsing and retrieval of image data", IEEE Transactions on Pattern Analysis and Machine Intelligence, Vol. 18, Issue. 8, pp. 837842, (1996).

[11] M.E. Mohamed, F. Ibrahima, B. S. Brahim, "Breast cancer diagnosis in digital mammogram using multiscale curvelet transform", Computerized Medical Imaging and Graphics, Vol. 34, Issue. 4, pp. 269-276, (2010).

[12] K. Peter, P. Nikolay, "Nonlinear Operator for Oriented Texture", IEEE Transactions on Image Processing, Vol. 8, Issue. 10, pp. 1395-1407, (1999).

[13] V. Vapnik, Statistical Learning Theory, Springer-Verlag, New York, NY, (1995).

[14] S. Yijun, T. Sinisa, G. Steve, "Local-Learning-Based Feature Selection for High-Dimensional Data Analysis", IEEE Transactions on Pattern Analysis and Machine Intelligence, Vol. 32, Issue. 9, pp. 1610-1626, (2010).

[15] Z. Yufeng, "Breast Cancer Detection with Gabor Features from Digital Mammograms", Algorithms, Vol. 3, No. 1, pp. 44-62, (2010).

[16] S. Yu, S. Shiguan, C. Xilin, G. Wen, "Hierarchical Ensemble of Global and Local Classifiers for Face Recognition”, IEEE Transactions on Image Processing, Vol. 18, Issue. 8, pp. 1885-1896, (2009).

[17] S. Zehan, B. Georg, M. Ronald, "Monocular Precrash Vehicle Detection: Features and Classifiers", IEEE Transactions on Image Processing, Vol. 15, Issue. 7, pp. 2019-2034, (2006). 